\title{
Systems neuroimmunology: a review of multiomics methodologies to characterize neuroimmunological interactions in spinal and cranial diseases
}

\author{
*Cameron Zamanian, BS, ${ }^{1,2}$ Archis R. Bhandarkar, BS, ${ }^{1,2,5}$ Dileep D. Monie, BA, ${ }^{2,4,5}$ \\ FM Moinuddin, PhD, ${ }^{1,2}$ Richard G. Vile, PhD, ${ }^{4}$ Alfredo Quiñones-Hinojosa, MD, ${ }^{3}$ and \\ Mohamad Bydon, MD1,2
}

${ }^{1}$ Neuro-Informatics Laboratory, ${ }^{2}$ Department of Neurosurgery, ${ }^{4}$ Department of Immunology, and ${ }^{5}$ Mayo Clinic Alix School of Medicine, Mayo Clinic, Rochester, Minnesota; and ${ }^{3}$ Department of Neurosurgery, Mayo Clinic, Jacksonville, Florida

\begin{abstract}
Neuroimmunology plays a critical role in our understanding of the pathophysiological processes that underlie a variety of diseases treated by neurosurgeons, including degenerative disc disease (DDD), glioblastoma (GBM), aneurysmal subarachnoid hemorrhage (aSAH), and others. Compared with traditional methods in neuroimmunology, which study one pathway or gene at a time, emerging multiomics methodologies allow for holistic interrogation of multiple immunesignaling pathways to test hypotheses and the effects of therapeutics at a systems level. In this review, the authors summarize key concepts for gathering and analyzing multiomics data so that neurosurgeons can contribute to the emerging field of systems neuroimmunology. Additionally, they describe 3 use cases, based on original research published by their group and others, that utilize transcriptomic, metabolomic, and proteomic analyses to study immune-signaling pathways in DDD, aSAH, and GBM. Through these use cases, techniques for performing machine learning and network-based analyses to generate new clinical insights from multiomics data are shared. The authors hope that neurosurgeons might use this review as a summary of common tools and principles in systems immunology to better engage in creating the immunotherapies of tomorrow.
\end{abstract}

https://thejns.org/doi/abs/10.3171/2021.11.FOCUS21571

KEYWORDS systems neuroimmunology; multiomics; transcriptomics; proteomics; metabolomics; degenerative disc disease; glioblastoma

$\mathrm{T}$ HE cellular microenvironment of the nervous system is composed of a dynamic milieu of vascular, immune, and neural cell types that interact with one another in a way that produces complex physiological responses. ${ }^{1}$ Neuroimmune interactions, in particular, play a critical role in both normal physiology and the pathophysiological processes that underlie cranial and spinal neurosurgical disease. In fact, the immune system has been found to have a key role in a variety of conditions treated by spine, tumor, and cerebrovascular neurosurgeons including 1) the immune-mediated progression of degenerative disc disease (DDD), ${ }^{2}$ ) the susceptibility of brain tumors like glioblastoma (GBM) ${ }^{3-6}$ to novel immunotherapies, and 3) the response of brain tissue to extravasated blood products during aneurysmal subarachnoid hemorrhage (aSAH)?
Systems neuroimmunology is an emerging framework for interrogating neuroimmunological interactions at a systems level, which can be used to study the immune processes that drive neurosurgical disease.$^{8-10}$ Systems neuroimmunology leverages multiomics data from genomic, proteomic, metabolomic, and other sources to arrive at a systems-level view of which genes, proteins, and metabolites of the immune system are active during healthy versus diseased states. The evolution of systems neuroimmunology into its modern form stems from advancements in molecular technologies, such as expression profiling, microchip array technology, immunological assay methods, and large-scale epitope screening. ${ }^{9}$ Neurosurgeons are particularly well poised to engage in systems neuroimmunology research, as their role is essential in gathering the tissue samples that generate multiomics data.

ABBREVIATIONS AF = annulus fibrosus; aSAH = aneurysmal subarachnoid hemorrhage; CCN1 = cellular communication network factor 1 ; DDD = degenerative disc disease; $\mathrm{DGE}=$ differential gene expression; $\mathrm{GBM}$ = glioblastoma; $\mathrm{HSV}=$ herpes simplex virus; $\mathrm{ICH}$ = intracerebral hemorrhage; IL = interleukin; IVD = intervertebral disc; MALDI-TOF = matrix-assisted laser desorption/ionization time of flight; NP = nucleus pulposus; OV = oncolytic virus; $\mathrm{PPI}=$ protein-protein interaction; scRNA-seq = singlecell RNA sequencing; TNF = tumor necrosis factor.

SUBMITTED September 24, 2021. ACCEPTED November 19, 2021.

INCLUDE WHEN CITING DOI: 10.3171/2021.11.FOCUS21571.

${ }^{*}$ C.Z., A.R.B., and D.D.M. contributed equally to this work. 


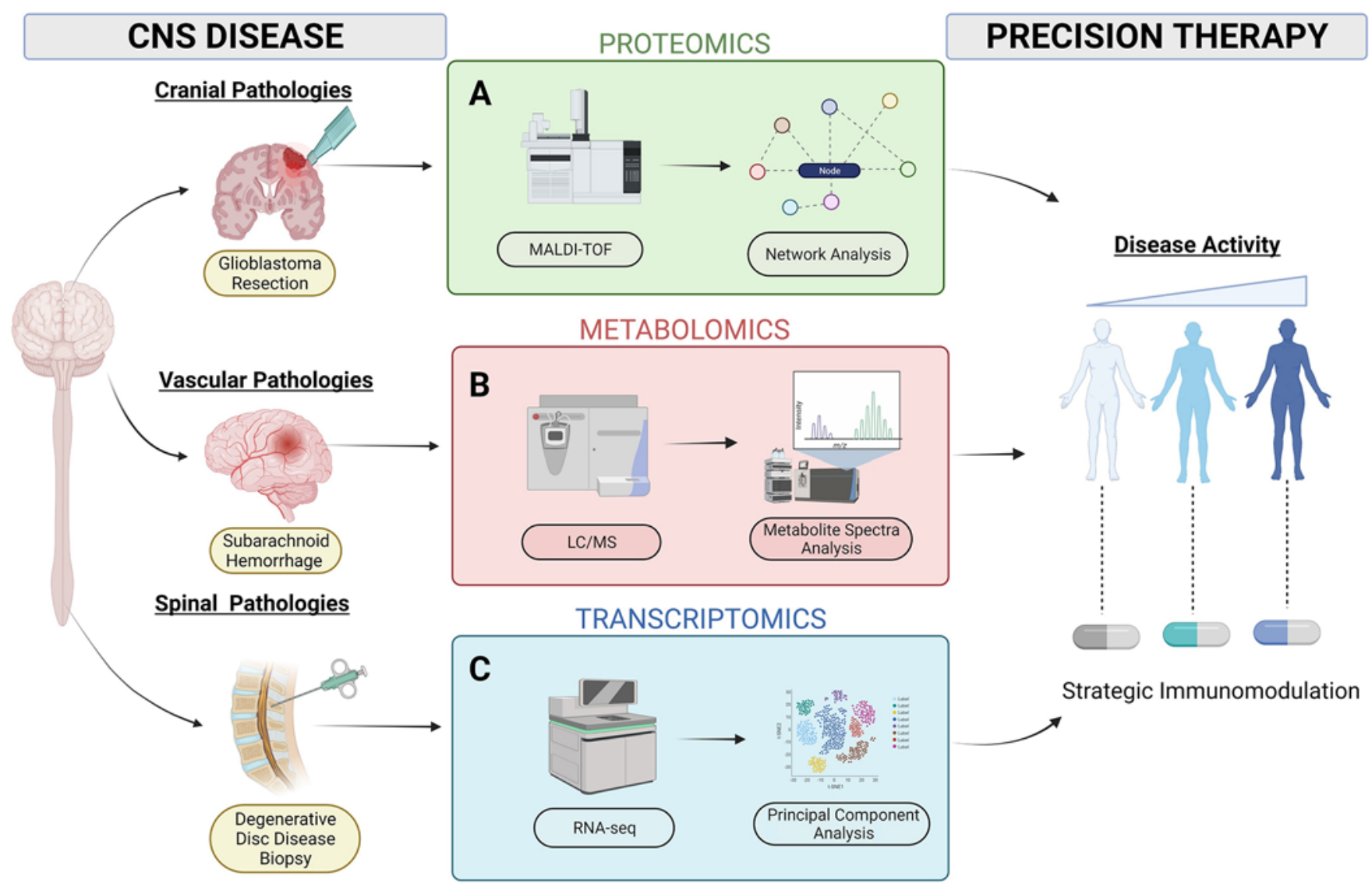

FIG. 1. A summary of multiomics approaches used to characterize neuroimmunological interactions in DDD and GBM. A: Proteomics can be used to identify susceptibilities of GBM to immunotherapy based on PPI network analysis. B: Metabolomics can identify metabolites that modulate immune interactions after aSAH. C: Transcriptomics can identify differentially expressed genes in immune-signaling pathways that drive DDD progression. LC/MS = liquid chromatography/mass spectometry; RNA-seq = RNA sequencing. Created with BioRender.com.

In this article, we hope to provide neurosurgeons with a practical guide for how they might use systems neuroimmunology in their practice to advance the collective understanding of neuroimmunological interactions in neurosurgical disease. We have selected 3 use cases that feature original research from our laboratories and others that apply systems neuroimmunology to the study of immunological interactions in spinal and cranial neurosurgical disease. These use cases each respectively demonstrate how multiomics data, including transcriptomic, metabolomic, and proteomic data, may be used to generate insights into how the immune system drives the progression of a broad variety of neurosurgical diseases including DDD, aSAH, and GBM (Fig. 1).

\section{Multiomics Technologies}

Systems neuroimmunology has successfully matured as a discipline due to technological advances in gathering multiomics data in a high-throughput fashion, as well as robust analysis pipelines for identifying alterations in immune signaling. In this section, we present some general principles related to generating, analyzing, and interpreting multiomics data so that neurosurgeons can better engage in this line of research.

\section{Generating Multiomics Data}

Systems neuroimmunology as applied to the study of neurosurgical pathologies relies on robust multiomics data sets generated by carefully curating tissue samples from healthy and diseased subjects. Neurosurgeons are essential to the process of collecting multiomics data for systems neuroimmunology research because they are directly involved in procedures that result in the excision of diseased tissue. For example, as described in use case 1, a standard lumbar discectomy and fusion can result in the excision of inflamed disc tissue, which can be used for systems neuroimmunology research. Several enabling technologies such as microarrays, RNA sequencing, and mass spectrometry then allow for multiomics data to be gathered from direct tissue biopsies, CSF, or blood (Table 1).

\section{Generating Genomic and Transcriptomic Data}

Genomic and transcriptomic data can be gathered from high-throughput technologies that quickly survey global gene expression patterns. Through microarray technology, global gene expression in tissue samples can be measured in parallel by thousands of genes and quantified to deduce signaling pathways and regulatory networks. ${ }^{11}$ The emergence of single-cell RNA sequencing (scRNA-seq) tech- 
TABLE 1. A summary of multiomics studies including the data source, enabling technologies, and insights created for neuroimmunology

\begin{tabular}{lcccc}
\hline Authors \& Year & -Omic Approach & Data Source & Enabling Technologies & Application in Neuroimmunology \\
\hline $\begin{array}{l}\text { Costantino et al., } \\
2017^{37}\end{array}$ & Genomics & Genes & DNA microarray & $\begin{array}{c}\text { Genome-wide association study in 906 pts, including 486 pts w/ } \\
\text { SpA; revealed association of SpA w/ MAPK14 }\end{array}$ \\
\hline $\begin{array}{l}\text { Bydon et al., } \\
2020^{2}\end{array}$ & Transcriptomics & RNA transcripts & RNA sequencing & $\begin{array}{c}\text { Transcriptomics data from IVD samples showed immune-signaling } \\
\text { pathways were upregulated in DDD }\end{array}$ \\
\hline $\begin{array}{l}\text { Ma et al., 20214 } \\
\text { Valentín-Guillama } \\
\text { et al., } 2018^{5}\end{array}$ & Proteomics & Proteins & MALDI-TOF & $\begin{array}{c}\text { A computational analysis of PPls in GBM cell lines identified oppor- } \\
\text { tunities to strategically improve immunotherapy design }\end{array}$ \\
\hline
\end{tabular}

gp120 = envelope glycoprotein 120; HPLC = high-performance liquid chromatography; pts = patients; SpA = spondyloarthritis.

nologies allows transcriptomic data to be gathered from individual cells. ${ }^{12,13}$ RNA sequencing requires single-cell suspensions to be obtained, which is typically achieved with high yield through mechanical disaggregation and enzymatic dissociation. Several technologies, such as flow cytometry, can be used to then isolate individual cells. After isolation, cells are lysed to extract RNA, which is subsequently amplified by polymerase chain reaction. ${ }^{12}$ It is important to assess the quality of the extracted RNA by measuring its integrity number. Given that RNA molecules are susceptible to degradation, the RNA integrity number is an important measure that can be used for quality control.$^{14}$ Before sequencing can be performed, the RNA must be converted into double-stranded complementary DNA, which can be achieved through poly-T oligo-attached magnetic beads. Sequencing can then be conducted, with reads mapped to a reference transcriptome or genome, and quantified to gene counts per gene or transcript. ${ }^{15} \mathrm{An}$ analysis of differential gene expression (DGE) enables quantification through computational statistical models to distinguish overlapping transcripts between samples. In use case 1 , we discuss a study that identified molecular regulators associated with DDD in an effort to establish promising candidates for therapeutic targets..$^{12,16}$

\section{Generating Proteomic and Metabolomic Data}

Mass spectrometry is the foundational technology for generating proteomic data and allows for characterization of proteins through analyzing their mass-to-charge ratio. ${ }^{17}$ Studying proteins in this fashion is a formidable task due to the fact that genes can generate multiple proteins through sequence polymorphisms, alternative splicing, and posttranscriptional modifications. These challenges have been overcome with several technological advances. ${ }^{17}$ For example, matrix-assisted laser desorption/ionization time of flight (MALDI-TOF), allows for the visualization of proteins in addition to their various proteoforms. ${ }^{18}$ With this technology, a matrix is used to coat the tissue under study, which aids in desorption and ionization of endogenous biomolecules during laser irradiation. Then, individual mass spectra are collected to generate signal intensity maps and ion images across the sample area. As thousands of these ion images are created through a single MALDI-TOF experiment, researchers can use top-down protein identification methods to gain molecular contexts of the tissue sample under study. ${ }^{18}$ In use case 2, we discuss how mass spectrometry is used to generate metabolomic data from tumor cell lines.

\section{Analyzing Multiomics Data}

Broadly, algorithms for analyzing multiomics data can be subdivided into clustering and network-based approaches. ${ }^{19}$

\section{Clustering Analysis}

Clustering algorithms are a type of unsupervised machine learning algorithm that can infer groups of similar samples based on a distance metric by using a weighted sum of expression values in multiomics data sets derived from different samples. ${ }^{20}$ Samples that have a distance apart from each other less than a given threshold are considered similar and are clustered into the same group. Common clustering algorithms used in neuroimmunology research include t-distributed stochastic neighbor embedding (t-SNE) and uniform manifold approximation and projection (UMAP) ${ }^{21}$ In Fig. 2A, we demonstrate an example of the hierarchical clustering that was employed in use case 1 to assess tissue-to-tissue and patient-to-patient variation in musculoskeletal tissue samples from patients with DDD.

\section{Network-Based Analysis}

Network-based approaches for analyzing multiomics data sets seek to identify dependent relationships and interactions between genes, RNA transcripts, and proteins. Such algorithms use metrics such as pairwise correlation, mutual information, or weighted coexpression to assess genes that are closely dependent on one another and to create a network. ${ }^{22}$ Some common network-based algorithms include NetDecoder (https://netdecoder.hms. harvard.edu), to derive context specificity, and the visualization packages Cytoscape (https://cytoscape.org) and NetworkX (https://networkx.org). ${ }^{21-23}$ In Fig. 2B, we show how a network-based algorithm was used to infer the immune-signaling pathways that were active in patients with DDD from use case 1.

For neurosurgeons just getting started with using such analytical approaches, there are several public multiomics 
A

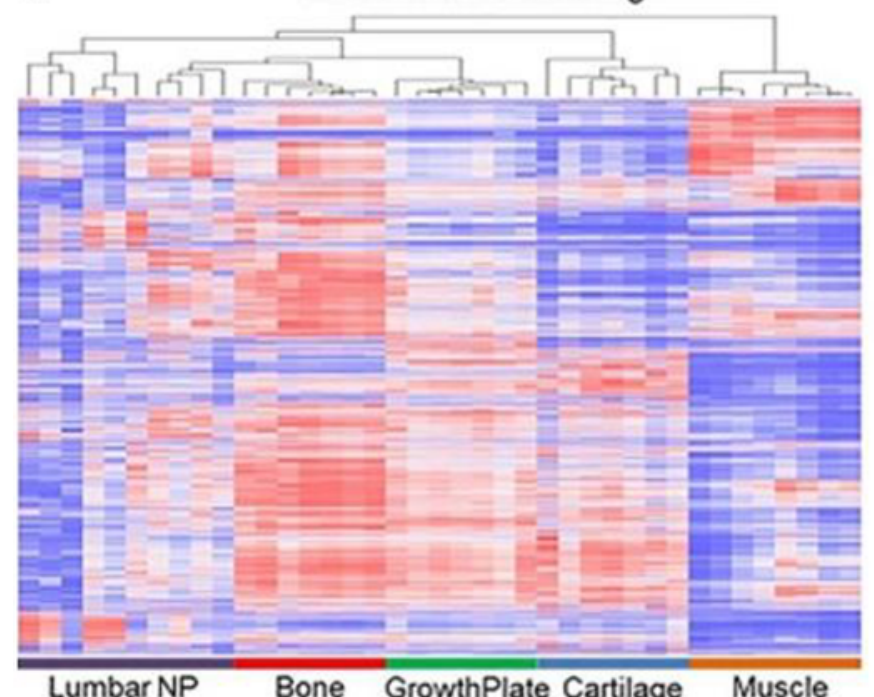

B

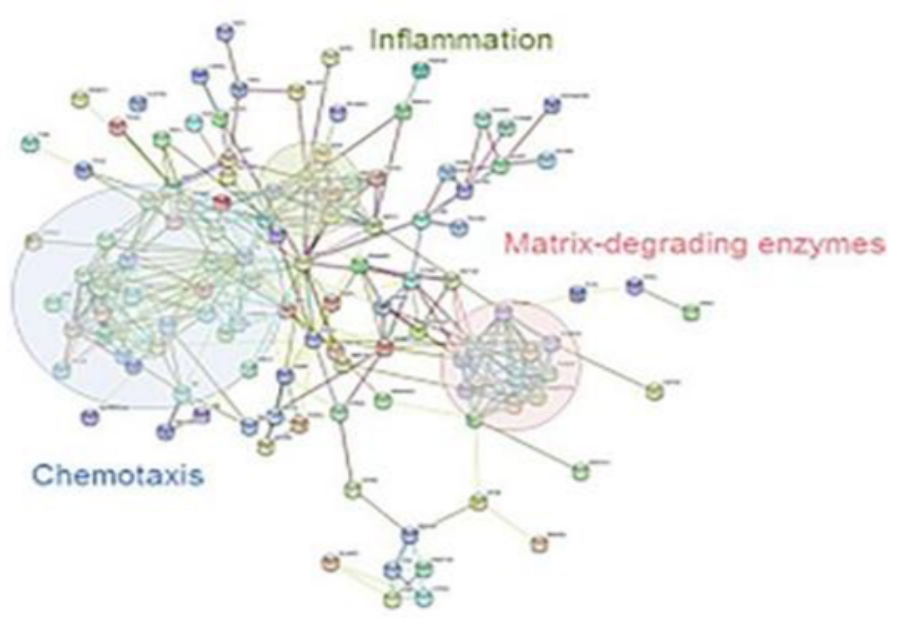

C

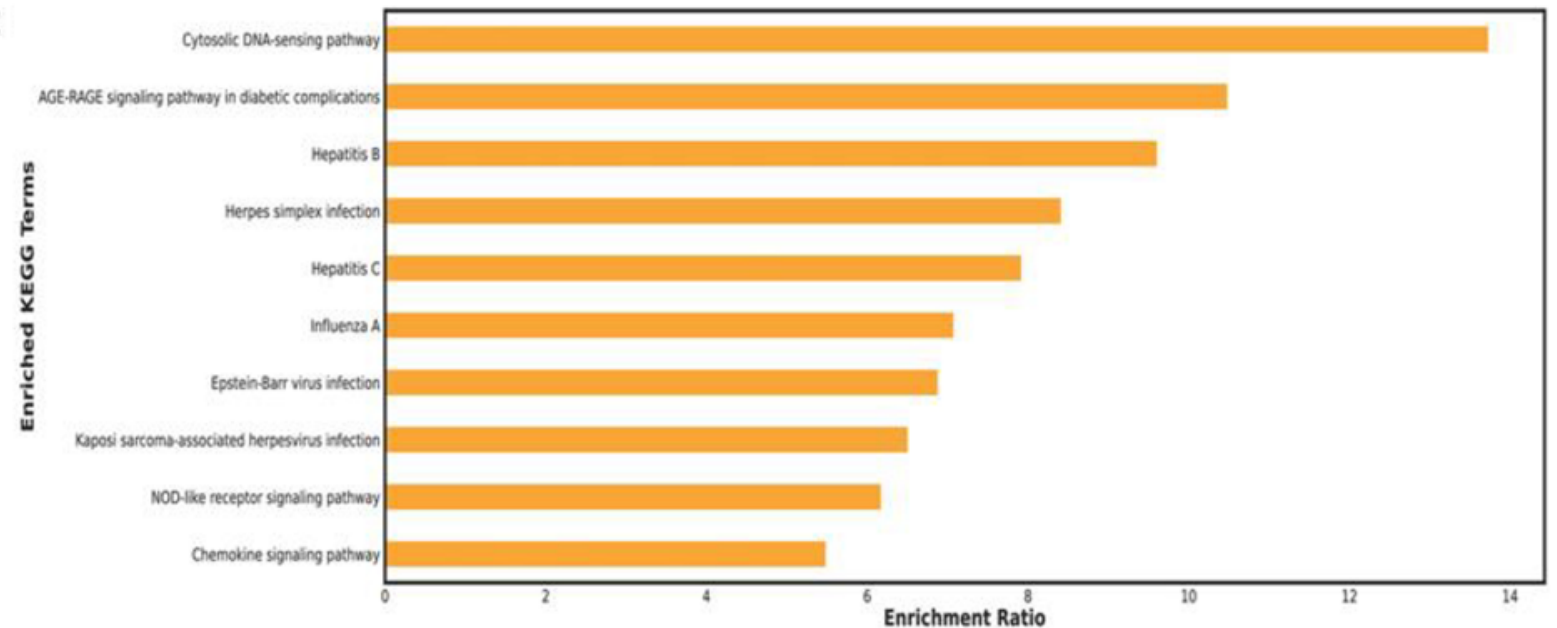

FIG. 2. Examples of different approaches employed to analyze multiomics data. A: Clustering methods can be used to assess patient-to-patient and tissue-to-tissue variation in musculoskeletal tissue samples from patients with DDD. B: Network methods can be used to assess immune-signaling pathways that are active in patients with DDD. C: Overrepresentation analyses can be used to detect signatures of resistance to OV therapy in GBM. AGE-RAGE = advanced glycation end products-receptor for advanced glycation end products; KEGG = Kyoto Encyclopedia of Genes and Genomes; NOD = nucleotide-binding oligomerization domain. Panels A and B reprinted from Gene, 750, Bydon M, Moinuddin FM, Yolcu YU, Wahood W, Alvi MA, Goyal A, Elminawy M,

Galeano-Garces C, Dudakovic A, Nassr A, Larson AN, van Wijnen AJ, Lumbar intervertebral disc mRNA sequencing identifies the regulatory pathway in patients with disc herniation and spondylolisthesis, 144634, Copyright 2020, with permission from Elsevier.

data resources that may be applied to neuroimmunology (Table 2). We advise, as a best practice, that different omics data sources should be integrated, which can serve to strengthen associations and reduce noise; in particular, similarity network fusion (SNF) is a commonly used method for integrating different omics data sources. ${ }^{23}$

\section{Generating New Insights From Multiomics Data}

Clinical insight generation is a critical component of multiomics pipelines in which neurosurgeons can actively participate. Often, the networks derived from the networkbased analyses described above can yield insights in and of themselves. For example, in use case 3, we have described how performing a network analysis on GBM mul- tiomics data elucidated mechanisms of resistance to oncolytic viral therapies. Additionally, we have described how the same network analysis allowed us to postulate that the measles virus was a more effective oncolytic viral vector compared with herpes simplex virus (HSV) for GBM with cellular communication network factor 1 (CCN1) resistance signatures (Fig. 2C).

Additionally, once an interaction network has been derived from multiomics data, novel clinical insights can be gathered by performing in silico simulations of network behavior. Network simulator tools like BioNSi (https:// bionsi.wixsite.com/bionsi) can estimate the behaviors of genes and proteins in a network once certain interactions are deleted or added. ${ }^{24}$ 
TABLE 2. Resources for public multiomics data sets

\begin{tabular}{lccc}
\hline \multicolumn{1}{c}{ Authors \& Year } & Data Resource & Multiomics Type & Description \\
\hline Edgar et al., 2002 & GEO & Genomics & A public, curated repository of microarray experiment data \\
\hline Darmanis et al., 201739 & GBMseq & Transcriptomics & $\begin{array}{c}\text { A public repository of scRNA-seq on 3,589 cells from GBM samples in a cohort } \\
\text { of 4 pts }\end{array}$ \\
\hline Xie et al., 201540 & HGCC & Transcriptomics & $\begin{array}{c}\text { An open resource, useful to both basic \& translational GBM research, containing } \\
\text { a biobank of 48 GBM cell lines \& an associated database containing high- } \\
\text { resolution molecular data }\end{array}$ \\
\hline Schmidt et al., 201841 & ProteomicsDB & Proteomics & A public, curated repository of proteomic data sets from a variety of host organisms \\
\hline Joshi et al., 201942 & TcellSubC & Proteomics & A public proteomic data set of 6,572 proteins in the human CD4+ T-cell proteome \\
\hline Sud et al., 2016 & Metabolomics Workbench & Metabolomics & A public, international repository for metabolomic data \\
\hline
\end{tabular}

GEO = Gene Expression Omnibus; HGCC = Human Glioblastoma Cell Culture; TcellSubC = T-Cell Subcellular Atlas.

\section{Systems Neuroimmunology and DDD Immunology Review in DDD}

DDD is a degenerative process affecting the mechanical integrity of intervertebral discs (IVDs) in the spine, which are composed of an inner nucleus pulposus (NP), outer annulus fibrosus (AF), and the bordering cartilaginous endplate. ${ }^{25}$ DDD is thought to be an age-related phenomenon that includes inflammation and cell apoptosis leading to decreased proteoglycan synthesis with resultant disc dehydration and loss of disc height. Multiomics can facilitate translational research to identify key genetic networks with sufficiently high levels of expression to make practical targets for genetic molecular therapy directed at ameliorating DDD.

Several studies in the neuroimmunology literature have elucidated the immune pathways that drive the progression of DDD. Initially, cells from the NP and AF secrete proinflammatory molecules that recruit immune cells to the IVD. ${ }^{26}$ Cytokines such as tumor necrosis factor (TNF) $-\alpha$, interleukin (IL)-1, IL-6, and IL-17 increase in concentration in the IVD, resulting in an inflammatory microenvironment. ${ }^{26}$ Such an environment reduces the structural integrity of the NP and AF, leading to herniation and annular tears. The degenerated IVDs subsequently amplify the inflammatory cascade through further production of TNF- $\alpha$, IL-1, IL-6, IL-17, interferon (IFN) $-\gamma$, and inflammatory mediators such as nitric oxide and prostaglandin $\mathrm{E} 2 . .^{27,28}$

It is imperative that neurosurgeons seeking therapeutic targets for DDD have a thorough understanding of the immunopathology for spine degeneration. In our first use case, we present work from our group that uses transcriptomic analysis to gain insight into DDD pathogenesis.

\section{Use Case 1: Transcriptomics to Characterize Inflammatory Pathways in DDD}

Our group published a study that aimed to identify immune-signaling pathways distinct to DDD progression. ${ }^{2}$ DDD may lead to spinal stenosis, disc herniation, and/ or degenerative spondylolisthesis, which may manifest in the patient as radiculopathy or myelopathy. To further enhance the understanding of disc herniation and degenerative spondylolisthesis pathogenesis, the transcriptome data of IVD samples from lumbar-degenerated discs were analyzed. Disc tissues were harvested intraoperatively and subsequently underwent RNA sequencing. ${ }^{2}$

A total of 33 adult patients who underwent lumbar discectomy to treat DDD were included in our study. During lumbar discectomy, NP samples from 33 adult patients were carefully dissected in the operating room by the primary surgeon. The harvested samples were then frozen in liquid nitrogen and stored until ready for processing. Of the 33 samples, only 10 samples met our threshold RNA integrity number necessary for next-generation sequencing.

The NP tissue biopsies were subsequently ground into powder and homogenized, then quantified with a spectrophotometer. Next-generation RNA sequencing was then performed to assess DGE. Computational analysis compared NP RNA sequencing with bone, cartilage, growth plate, and muscle tissue. ${ }^{2}$

When we compared transcriptomic data from NP samples with other musculoskeletal tissues in patients with DDD, we were able to identify several immune-signaling pathways that were upregulated. When comparing the NP samples with other musculoskeletal tissues, 702 genes demonstrating significant upregulation and 3734 genes with significant downregulation were found. To better understand the unique regulatory pathway for disc herniation and degenerative spondylolisthesis, the DGE profile revealed 1514 upregulated and 281 downregulated mRNA molecules. Furthermore, STRING analysis (https://stringdb.org) demonstrated differentially expressed genes for each gene ontology term from different types of networks, with distinct cellular functions for each biomedical condition. Specifically, we found several differentially expressed genes in chemotactic signaling (CXCL10, CXCL11, ILIRL2, and IL-6) and matrix-degrading pathways (MMP16, ADAMTSL1, ADAMTSL5, ADAMTSL8, ADAMTSL12, and ADAMTSL15). Neurosurgeons can leverage these identified gene regulatory networks, or conduct similar transcriptomic analysis, to strategically engineer novel pharmacological approaches for the treatment of lumbar DDD and other degenerative diseases.

\section{Systems Neuroimmunology and aSAH Immunology Review in aSAH}

Extravasated blood products that are released after intracerebral hemorrhage $(\mathrm{ICH})$ or aSAH are known trig- 
gers of inflammation in the brain. ${ }^{29,30}$ While primary injury, which occurs within hours after $\mathrm{ICH}$, is related to the mechanical distortion of brain tissue due to hematoma expansion, secondary injury, which occurs on a much longer time scale after the initial insult, is often mediated by immune signaling. Red blood cell lysis occurs approximately 24 hours after an initial ICH and has been demonstrated to activate resident microglia and astrocytes. These neuroglia in turn release cytokines such as TNF- $\alpha$ and IL-1 $\beta$, which recruit circulating immune cells, including neutrophils and macrophages. ${ }^{29}$ In aSAH, the pathway toward neutrophil recruitment is similar, and cytokines like IL-6, IL-1 $\alpha$, IL-1 $\beta$, IL-8, and TNF- $\alpha$ have been implicated as contributors to cerebral vasospasm. ${ }^{30}$

High-throughput methods from multiomics are enabling researchers to interrogate changes in the CSF of patients with aSAH and $\mathrm{ICH}$. Here, we summarize recent work from Koch et al. ${ }^{7}$ that used metabolomics and machine learning to study biomarkers in the CSF of patients with aSAH.

\section{Use Case 2: Metabolomics to Evaluate Outcomes After aSAH}

In their study, Koch et al. used metabolomic data derived from the CSF samples of 81 patients with aSAH to identify key metabolites in an immune-signaling pathway that were predictive of poor outcomes after aSAH. ${ }^{7}$ CSF samples were obtained from unclamped external ventricular drains at 3 different time points (0-5 days, 6-10 days, and 11-15 days) after admission. CSF samples were additionally obtained in a control cohort of 16 patients with nonruptured cerebral aneurysms through either a lumbar drain or lumbar puncture.

Several steps were taken to prepare CSF samples for metabolomic analysis. Samples were centrifuged to pellet out cellular material, and the resulting supernatant was aliquoted and treated with cetonitrile/methanol (3:1) with citrulline-d8, in order to prepare the samples for processing through a high-performance liquid chromatography system. Mass spectrometry was then used to quantitatively measure the concentrations of a total of 138 metabolites.

To process the metabolomic data, the authors used machine learning models to identify which metabolites were most strongly associated with modified Rankin Scale scores at discharge and at 90 days after discharge. They found that the concentrations of key metabolites that alter the nitric oxide signaling pathway, including symmetric dimethylarginine, dimethylguanidine valeric acid, and ornithine, were associated with a poor modified Rankin Scale score at discharge and at 90 days.

The methodology of Koch et al. provides an example of how multiomics can elucidate how key metabolites that regulate immune-signaling pathways can also shape outcomes after neurosurgical disease. Their findings provide key biomarkers for predicting outcomes and point to potential drug targets for modulating the immune response after aSAH. Neurosurgeons may use a similar multiomics workflow as that of Koch et al. to study immune-signaling pathways that are upregulated in the CSF samples of patients with epilepsy, spinal cord injury, and other neurosurgical diseases.

\section{Systems Neuroimmunology and GBM Immunology Review in GBM}

GBM is an aggressive brain tumor characterized by significant cellular and molecular heterogeneity, as well as complex interactions with the host immune system. ${ }^{31}$ Recent work characterizing the GBM microenvironment has led to the discovery of functionally distinct immune cells in tumors, including tumor-infiltrating dendritic cells, regulatory $\mathrm{T}$ cells, cytotoxic $\mathrm{T}$ cells, and tumor-associated macrophages, each of which uniquely shape the response to immunotherapies. ${ }^{32}$ For example, regulatory $\mathrm{T}$ cells recruited by GBM cells can promote tumorigenesis by suppressing the cytotoxic effect of tumor-infiltrating dendritic cells and cytotoxic $\mathrm{T}$ cells. ${ }^{33}$ Given how complex these interactions between immune cells and GBM cells are, there has been an increasing appreciation of the need for systems-based approaches to develop new immunotherapies for GBM. ${ }^{34}$

Herein, we present use case 3, our second use case based on original work that uses systems neuroimmunology to better understand how the immune system interacts with GBM, in which we aimed to determine potential therapeutic targets for HSV-1-derived oncolytic viruses (OVs) to treat GBM. This use case underscores how proteomic data analysis can generate insights about GBM neuroimmunology.

\section{Use Case 3: Protein-Protein Interaction Analysis to Identify Tumor Susceptibility to Immunotherapies}

Immunotherapies use the host immune system to target GBM cells and are broadly divided into 2 categories: passive and active. ${ }^{35}$ Passive immunotherapies, such as monoclonal antibodies, require continuous administration over time as they produce short-lived, yet specific, immune responses. On the other hand, active immunotherapies, including OVs, are a direct stimulation of an immune response with long-term effects. OV immunotherapy for cancer dates back to the 1990s, when HSV-1 was engineered to selectively replicate and target tumor cells. Since then, active immunotherapies have made significant strides and are the focal point of many clinical trials. ${ }^{35}$

Our team previously published a study ${ }^{3}$ that aimed to further understand HSV-1 OV resistance by investigating the role of CCN1 in the GBM intracellular state. Found in most GBM microenvironments, CCN1 expression has been shown to predict resistance to OV. This work builds on a study by Haseley et al., ${ }^{36}$ which found that CCN1 binds and activates cell-surface integrin $\alpha 6 \beta 1$, promoting an antiviral and protumor state. GBM with high concentrations of $\mathrm{CCN} 1$ (CCN1 $^{\text {high }}$ ) has demonstrated worse rates of overall and progression-free survival. ${ }^{3}$

To further understand the downstream effect of CCN1, transcriptomic data of LN229 GBM cell lines were mapped to known protein-protein interactions (PPIs) in the iRefIndex (https://irefindex.vib.be) database. These PPIs were synthesized into networks and analyzed in NetDecoder to compare $\mathrm{CCN} 1^{\text {high }}$ with $\mathrm{CCN} 1^{\text {low }} \mathrm{GBM}$ phenotypes in order to elucidate critical differences between the two states. High-impact genes, network routers, key targets, and CCN1-specific edges were identified through informa- 
tion flow analysis. We further compared these cell states through network and motif modeling, overrepresentation analysis, and assessment of gene dependencies. ${ }^{3}$

After studying differential edge flows, we identified 39 nodes and 12 binary edges that may potentially determine susceptibility of CCN1 ${ }^{\text {high }}$ GBM to OV. Furthermore, CCN1 ${ }^{\text {high }}$ states were shown to exploit IDH1 and TP53 and increase dependency on RPL6, HUWE1, and COPS5. Our findings were reproduced in 65 other GBM cell lines and 174 clinical sample data sets from patients with GBM for validation. $^{3}$

Our study identified novel pathways, proteins, and interactions critical to the $\mathrm{CCN} 1^{\text {high }}$ GBM phenotype. Conducting a generalized network model and systems-level analysis allowed us to identify several innate immune pathways in GBM that CCN1 leverages to disrupt HSV-1 OV immunotherapy. Additionally, we found opportunities to strategically improve the HSV-1 OV design. For example, because measles virus is not present in the resistance signatures that we identified through overexpression analysis (Fig. 2C), we postulated that measles virus may be a better vector than HSV-1 for GBM treatment. ${ }^{3}$ In a similar vein, other neurosurgeons can use this approach to design more effective immunotherapies for GBMs and other CNS malignancies.

\section{Conclusions}

Recent evidence has demonstrated that neuroimmunemediated processes have an essential role in both the normal physiology of the nervous system and the pathophysiological processes that drive neurosurgical disease. The study of the multiome, which integrates genomic, transcriptomic, proteomic, and other omics data sources, is driving new discoveries in neuroimmunology. Compared with traditional approaches in neuroimmunology, multiomics methods allow for holistic interrogation of multiple immune-signaling pathways at a systems level. In this review, we described 3 use cases in which multiomics approaches were used to investigate the neuroimmunology of different conditions treated by neurosurgeons, including DDD, GBM, and aSAH. We hope that the methods outlined here will catalyze further research using systems immunology and empower neurosurgeons to generate future discoveries in neuroimmunology.

\section{Acknowledgments}

D.D.M. was supported by an individual fellowship from the National Cancer Institute (F30 CA250122), an institutional training grant from the National Institute of General Medical Sciences (T32 GM65841), the Mayo Clinic Medical Scientist Training Program, and the Mayo Clinic Center for Regenerative Medicine. A.Q.H. was supported by research grants from the National Institutes of Health (R01 CA216855R01 CA195503, R01 CA200399, R01 CA216855, and R33 CA240181).

\section{References}

1. Daneman R, Prat A. The blood-brain barrier. Cold Spring Harb Perspect Biol. 2015;7(1):a020412.

2. Bydon M, Moinuddin FM, Yolcu YU, et al. Lumbar intervertebral disc mRNA sequencing identifies the regulatory pathway in patients with disc herniation and spondylolisthesis. Gene. 2020;750:144634.
3. Monie DD, Correia C, Zhang C, Ung CY, Vile RG, Li H. Modular network mechanism of CCN1-associated resistance to HSV-1-derived oncolytic immunovirotherapies for glioblastomas. Sci Rep. 2021;11(1):11198.

4. Ma T, Hu C, Lal B, et al. Reprogramming transcription factors Oct4 and Sox 2 induce a BRD-dependent immunosuppressive transcriptome in GBM-propagating cells. Cancer Res. 2021;81(9):2457-2469.

5. Valentín-Guillama G, López S, Kucheryavykh YV, et al. HIV-1 Envelope protein gp120 promotes proliferation and the activation of glycolysis in glioma cell. Cancers (Basel). 2018; 10(9):E301.

6. Al-Kharboosh R, ReFaey K, Lara-Velazquez M, Grewal SS, Imitola J, Quiñones-Hinojosa A. Inflammatory mediators in glioma microenvironment play a dual role in gliomagenesis and mesenchymal stem cell homing: implication for cellular therapy. Mayo Clin Proc Innov Qual Outcomes. 2020;4(4): 443-459.

7. Koch M, Acharjee A, Ament Z, et al. Machine learning-driven metabolomic evaluation of cerebrospinal fluid: insights into poor outcomes after aneurysmal subarachnoid hemorrhage. Neurosurgery. 2021;88(5):1003-1011.

8. Davis MM, Tato CM, Furman D. Systems immunology: just getting started. Nat Immunol. 2017;18(7):725-732.

9. Khan-Farooqi HR, Prins RM, Liau LM. Tumor immunology, immunomics and targeted immunotherapy for central nervous system malignancies. Neurol Res. 2005;27(7):692-702.

10. Villani AC, Sarkizova S, Hacohen N. Systems immunology: learning the rules of the immune system. Annu Rev Immunol. 2018;36:813-842.

11. Braga-Neto UM, Marques ETA Jr. From functional genomics to functional immunomics: new challenges, old problems, big rewards. PLoS Comput Biol. 2006;2(7):e81.

12. Stark R, Grzelak M, Hadfield J. RNA sequencing: the teenage years. Nat Rev Genet. 2019;20(11):631-656.

13. Liang Q, Dharmat R, Owen L, et al. Single-nuclei RNA-seq on human retinal tissue provides improved transcriptome profiling. Nat Commun. 2019;10(1):5743.

14. Schroeder A, Mueller O, Stocker S, et al. The RIN: an RNA integrity number for assigning integrity values to RNA measurements. BMC Mol Biol. 2006;7:3.

15. Owens NDL, De Domenico E, Gilchrist MJ. An RNA-Seq protocol for differential expression analysis. Cold Spring Harb Protoc. 2019;2019(6).

16. Hasin Y, Seldin M, Lusis A. Multi-omics approaches to disease. Genome Biol. 2017;18(1):83.

17. Domon B, Aebersold R. Mass spectrometry and protein analysis. Science. 2006;312(5771):212-217.

18. Ryan DJ, Spraggins JM, Caprioli RM. Protein identification strategies in MALDI imaging mass spectrometry: a brief review. Curr Opin Chem Biol. 2019;48:64-72.

19. Subramanian I, Verma S, Kumar S, Jere A, Anamika K. Multi-omics data integration, interpretation, and its application. Bioinform Biol Insights. 2020;14:1177932219899051.

20. Rappoport N, Shamir R. Multi-omic and multi-view clustering algorithms: review and cancer benchmark. Nucleic Acids Res. 2018;46(20):10546-10562.

21. Kobak D, Berens P. The art of using t-SNE for single-cell transcriptomics. Nat Commun. 2019;10(1):5416.

22. Yan J, Risacher SL, Shen L, Saykin AJ. Network approaches to systems biology analysis of complex disease: integrative methods for multi-omics data. Brief Bioinform. 2018;19(6): $1370-1381$

23. Wang B, Mezlini AM, Demir F, et al. Similarity network fusion for aggregating data types on a genomic scale. Nat Methods. 2014;11(3):333-337.

24. Rubinstein A, Bracha N, Rudner L, Zucker N, Sloin HE, Chor B. BioNSi: a discrete Biological Network Simulator tool. $J$ Proteome Res. 2016;15(8):2871-2880. 
25. Leone G, Torricelli P, Chiumiento A, Facchini A, Barbucci $\mathrm{R}$. Amidic alginate hydrogel for nucleus pulposus replacement. J Biomed Mater Res A. 2008;84(2):391-401.

26. Livshits G, Kalinkovich A. Hierarchical, imbalanced proinflammatory cytokine networks govern the pathogenesis of chronic arthropathies. Osteoarthritis Cartilage. 2018;26(1): 7-17.

27. Navone SE, Marfia G, Giannoni A, et al. Inflammatory mediators and signalling pathways controlling intervertebral disc degeneration. Histol Histopathol. 2017;32(6):523-542.

28. Weber KT, Alipui DO, Sison CP, et al. Serum levels of the proinflammatory cytokine interleukin- 6 vary based on diagnoses in individuals with lumbar intervertebral disc diseases. Arthritis Res Ther. 2016;18:3.

29. Zhou Y, Wang Y, Wang J, Anne Stetler R, Yang QW. Inflammation in intracerebral hemorrhage: from mechanisms to clinical translation. Prog Neurobiol. 2014;115:25-44.

30. Schneider UC, Xu R, Vajkoczy P. Inflammatory events following subarachnoid hemorrhage (SAH). Curr Neuropharmacol. 2018;16(9):1385-1395.

31. Wang Z, Sun D, Chen YJ, et al. Cell lineage-based stratification for glioblastoma. Cancer Cell. 2020;38(3):366-379.e8.

32. Pombo Antunes AR, Scheyltjens I, Duerinck J, Neyns B, Movahedi K, Van Ginderachter JA. Understanding the glioblastoma immune microenvironment as basis for the development of new immunotherapeutic strategies. eLife. 2020;9:e52176.

33. DeCordova S, Shastri A, Tsolaki AG, et al. Molecular heterogeneity and immunosuppressive microenvironment in glioblastoma. Front Immunol. 2020;11:1402.

34. Monie DD, Bhandarkar AR, Parney IF, et al. Synthetic and systems biology principles in the design of programmable oncolytic virus immunotherapies for glioblastoma. Neurosurg Focus. 2021;50(2):E10.

35. Abbott M, Ustoyev Y. Cancer and the immune system: the history and background of immunotherapy. Semin Oncol Nurs. 2019;35(5):150923.

36. Haseley A, Boone S, Wojton J, et al. Extracellular matrix protein CCN1 limits oncolytic efficacy in glioma. Cancer Res. 2012;72(6):1353-1362.

37. Costantino F, Talpin A, Said-Nahal R, et al. A family-based genome-wide association study reveals an association of spondyloarthritis with MAPK14. Ann Rheum Dis. 2017;76(1): 310-314.
38. Edgar R, Domrachev M, Lash AE. Gene Expression Omnibus: NCBI gene expression and hybridization array data repository. Nucleic Acids Res. 2002;30(1):207-210.

39. Darmanis S, Sloan SA, Croote D, et al. Single-cell RNA-Seq analysis of infiltrating neoplastic cells at the migrating front of human glioblastoma. Cell Rep. 2017;21(5):1399-1410.

40. Xie Y, Bergström T, Jiang Y, et al. The human glioblastoma cell culture resource: validated cell models representing all molecular subtypes. EBioMedicine. 2015;2(10):1351-1363.

41. Schmidt T, Samaras P, Frejno M, et al. ProteomicsDB. Nucleic Acids Res. 2018;46(D1):D1271-D1281.

42. Joshi RN, Stadler C, Lehmann R, et al. TcellSubC: an atlas of the subcellular proteome of human T cells. Front Immunol. 2019;10:2708.

43. Sud M, Fahy E, Cotter D, et al. Metabolomics Workbench: an international repository for metabolomics data and metadata, metabolite standards, protocols, tutorials and training, and analysis tools. Nucleic Acids Res. 2016;44(D1):D463-D470.

\section{Disclosures}

The authors report no conflict of interest concerning the materials or methods used in this study or the findings specified in this paper.

\section{Author Contributions}

Conception and design: Zamanian, Bhandarkar, Monie. Acquisition of data: Zamanian, Bhandarkar, Monie. Analysis and interpretation of data: Zamanian, Bhandarkar, Monie, Moinuddin, Vile. Drafting the article: Zamanian, Bhandarkar, Monie, Moinuddin, Vile, Quiñones-Hinojosa. Critically revising the article: Zamanian, Bhandarkar, Monie, Moinuddin, Vile, Quiñones-Hinojosa. Reviewed submitted version of manuscript: Zamanian, Bhandarkar, Monie. Approved the final version of the manuscript on behalf of all authors: Bydon. Statistical analysis: Monie. Study supervision: Bydon.

\section{Correspondence}

Mohamad Bydon: Mayo Clinic, Rochester, MN. bydon. mohamad@mayo.edu. 